\title{
TRATAMIENTO DE UN AGUA RESIDUAL INDUSTRIAL A TEMPERATURA PSICROFÍLICA CON UN REACTOR UASB
}

Treatment of industrial residual water at psychrophilic temperature with a UASB reactor

\section{Adriana JACOBO LÓPEZ ${ }^{1}$, Mario ESPARZA SOTO ${ }^{2}$, Mercedes LUCERO CHÁVEZ $^{2 *}$ y Cheikh FALL ${ }^{2}$}

${ }^{1}$ Facultad de Ingeniería, Universidad Autónoma del Estado de México, Cerro Coatepec S/N, Ciudad Universitaria, 50100 Toluca, Estado de México

${ }^{2}$ Instituto Interamericano de Tecnología y Ciencias del Agua, Universidad Autónoma del Estado de México, Carretera Toluca - Ixtlahuaca Km 14.5, Toluca, Estado de México

*Autora para correspondencia: mluceroc@uaemex.mx

(Recibido: enero 2018; aceptado: noviembre 2018)

Palabras clave: baja temperatura, carga orgánica volumétrica, biogás, rendimiento celular

\section{RESUMEN}

En el presente trabajo se operó un reactor anaerobio de flujo ascendente (UASB, por sus siglas en inglés) a escala de laboratorio con un tiempo de retención hidráulico $(6 \mathrm{~h})$ y a temperatura psicrofílica $\left(<20^{\circ} \mathrm{C}\right)$. En el reactor UASB se trató agua residual de una industria chocolatera con dos cargas orgánicas volumétricas aplicadas $\left(\mathrm{COV}_{\mathrm{apl}}\right)$. Cada $\mathrm{COV}_{\text {apl }}$ correspondió a una etapa experimental: la etapa experimental I con $1.8 \pm$ $0.8 \mathrm{~kg}$ de demanda química de oxígeno soluble $\left(\left[\mathrm{DQO}_{\mathrm{s}}\right] / \mathrm{m}^{3} / \mathrm{d}\right)$ y la etapa experimental II con $3.5 \pm 0.9 \mathrm{~kg} \mathrm{DQO} / \mathrm{m}^{3} / \mathrm{d}$. El objetivo fue evaluar la eficiencia de remoción de la $\mathrm{DQO}_{\mathrm{s}}(\mathrm{ER})$, la producción de biogás $(\mathrm{PB})$ y el rendimiento celular experimental $\left(Y_{\exp }\right)$ en un reactor UASB a escala de laboratorio. Los parámetros de control monitoreados fueron $\mathrm{pH}$, temperatura y flujo. La alcalinidad, ácidos grasos volátiles y $\mathrm{DQO}_{\mathrm{s}}$ se analizaron en el influente y efluente, mientras que los sólidos suspendidos se analizaron dentro del reactor y en el efluente. Durante el estado estacionario de las dos etapas experimentales la ER fue mayor al $90 \%$. La elevada ER obtenida mostró que en el reactor UASB se trató eficientemente el agua residual industrial a baja temperatura con las $\mathrm{COV}_{\text {apl. }} \mathrm{La} \mathrm{PB}$ se incrementó cuando la $\mathrm{COV}_{\text {apl }}$ aumentó $(1.6 \pm 0.8$ y $3.5 \pm$ $1.3 \mathrm{~L} / \mathrm{d}$ con la $\mathrm{COV}_{\text {apl }}$ de 1.8 y $3.5 \mathrm{~kg} \mathrm{DQO}_{\mathrm{s}} / \mathrm{m}^{3} / \mathrm{d}$, respetivamente $\left[1 \mathrm{~atm}, 273^{\circ} \mathrm{K}\right]$ ). El $Y_{\exp }$ de la etapa experimental II fue alto $\left(0.11 \mathrm{~kg} \mathrm{SSV} / \mathrm{kg} \mathrm{DQO}_{\mathrm{rem}}\right)$, lo cual pudo deberse a que la temperatura psicrofílica influyó sobre la tasa de crecimiento de los microorganismos anaerobios.

Key words: low temperature, organic volumetric load, biogas and cell yield

\begin{abstract}
In the present work, a laboratory scale anaerobic upflow reactor (UASB) was operated with a hydraulic retention time $(6 \mathrm{~h})$ and at psychrophilic temperature $\left(<20^{\circ} \mathrm{C}\right)$. The UASB reactor treated wastewater from a chocolate industry with two applied organic volumetric loads $\left(\mathrm{OLR}_{\mathrm{apl}}\right)$. Each $\mathrm{OLR}_{\mathrm{apl}}$ corresponded to an experimental stage: experimental stage I with $1.8 \pm 0.8 \mathrm{~kg}$ soluble oxygen chemical demand $\left(\left[\mathrm{DQO}_{\mathrm{s}}\right] / \mathrm{m}^{3} / \mathrm{d}\right.$ ) and experimental stage II with $3.5 \pm 0.9 \mathrm{~kg} \mathrm{COD}_{\mathrm{s}} / \mathrm{m}^{3} / \mathrm{d}$. The objective of this research
\end{abstract}


was to evaluate the removal efficiency of CODs (RE), biogas production (BP) and experimental cell yield $\left(Y_{\exp }\right)$ within the laboratory scale UASB reactor. The control parameters monitored were $\mathrm{pH}$, temperature and flow. Alkalinity, volatile fatty acids and $\mathrm{COD}_{\mathrm{s}}$ were analyzed in the influent and effluent, while suspended solids were analyzed inside the reactor and in the effluent. The RE during the steady state of the two experimental stages was greater than $90 \%$. The high RE obtained showed that the UASB reactor efficiently treated the industrial wastewater at low temperature with the OLR $\mathrm{apl}_{\text {ap }}$ BP increased when OLR $\mathrm{apl}_{\text {increased }}(1.6 \pm 0.8$ and $3.5 \pm 1.3 \mathrm{~L} / \mathrm{d}$ of $\mathrm{OLR}_{\mathrm{apl}}$ of 1.8 and $3.5 \mathrm{~kg} \mathrm{COD} / \mathrm{m}^{3} / \mathrm{d}$, respectively [1 atm, $\left.\left.273 \mathrm{~K}\right]\right)$. The $Y_{\exp }$ of experimental stage II was high $\left(0.11 \mathrm{~kg} \mathrm{SSV} / \mathrm{kg} \mathrm{COD}_{\mathrm{rem}}\right)$. The high $Y_{\exp }$ of experimental stage II could be due to the influence of psychrophilic temperature in the growth rate of anaerobic microorganisms.

\section{INTRODUCCIÓN}

El tratamiento del agua residual con sistemas biológicos anaerobios se ha incrementado durante los últimos 30 años en comparación con los tratamientos aerobios, debido principalmente a su bajo requerimiento energético, la escasa formación de lodo residual y la producción de metano que representa una potencial fuente de energía (Parawira et al. 2006).

En las últimas tres décadas se han desarrollado reactores anaerobios de alta tasa, tales como el proceso anaerobio de contacto (ACP, por sus siglas en inglés), filtros anaerobios (AF, por sus siglas en inglés), el reactor anaerobio de flujo ascendente y manto de lodo (UASB, por sus siglas en inglés), el reactor de lecho fluidizado ( $\mathrm{FB}$, por sus siglas en inglés), el reactor de cama de lodo granular expandida (EGSB, por sus siglas en inglés) y el reactor anaerobio con deflectores (ABR, por sus siglas en inglés).

El reactor UASB de alta tasa es el más utilizado en el tratamiento del agua residual. Al comienzo de 2007, cerca 1750 reactores UASB a gran escala se pusieron en marcha alrededor del mundo para tratar agua residual agroindustrial, química y municipal (Henze et al. 2008).

El éxito del reactor UASB se puede atribuir a su capacidad para retener altas concentraciones de lodo en condiciones anaerobias; lo anterior permite tratar altas cargas orgánicas volumétricas aplicadas $\left(\mathrm{COV}_{\text {apl }}\right)$, mientras mantiene un tiempo de retención celular (TRC) largo y un tiempo de retención hidráulico (TRH) relativamente corto (Henze et al. 2008).

La temperatura de operación de reactores biológicos influye directamente en dos aspectos de las reacciones biológicas de las bacterias presentes: la velocidad de las reacciones catalizadas enzimáticamente y la velocidad de difusión del sustrato a las células. La mayoría de los coeficientes de velocidad de reacción aumentan a medida que se incrementa la temperatura, pero eventualmente disminuyen a medida que la temperatura empieza a inactivar las enzimas de las células.

La baja temperatura dificulta el transporte de sustrato dentro de las células (Grady et al. 1999) e influye en las actividades metabólicas de la población microbiana, mostrando un efecto negativo en la tasa de transferencia de gas, en las características de sedimentación de los sólidos biológicos (Tchobanoglous et al. 2014) y en las propiedades fisicoquímicas del agua residual (Lettinga et al. 2001).

La temperatura recomendable de operación en reactores anaerobios es de 25 a $35{ }^{\circ} \mathrm{C}$ para obtener las velocidades de las reacciones biológicas óptimas y proporcionar un tratamiento más estable (Tchobanoglous et al. 2014). Por lo anterior, los trabajos de investigación con reactores UASB comúnmente se han realizado a temperaturas $>30^{\circ} \mathrm{C}$ (Atuanya y Chakrabarti 2004, Chou et al. 2004, Diamantis y Aivasidis 2007, Sponza y Uluköy 2008, Yetilmezsoy y Sakar 2008, Nacheva et al. 2009, El-Sheikh et al. 2011, Senthilkumar et al. 2011, Wang et al. 2011, Wang y Han 2012, Qiu et al. 2013, Hinken et al. 2014, Li et al. 2015a, b, Lu et al. 2015).

La operación de reactores UASB a temperatura mesofílica representa un costo energético considerable y sobre todo en regiones de clima frío, como la ciudad de Toluca, Estado de México, donde la temperatura del agua varía de 12 a $20^{\circ} \mathrm{C}$, dependiendo de la estación del año.

La mayoría de los trabajos encontrados en la literatura realizados en reactores UASB a baja temperatura $\left(<20^{\circ} \mathrm{C}\right)$ emplearon agua residual sintética o municipal (Singh y Viraraghavan 2002, Mahmoud et al. 2004, Álvarez et al. 2006, Akila y Chandra 2007, Bandara et al. 2011, 2012, Turkdogan-Aydino et al. 2011, Farajzadehha et al. 2012, Donoso-Bravo et al. 2013, Zhang et al. 2013, Rizvi et al. 2015, Saha et al. 2015). Sin embargo, se han realizado 
pocos estudios sobre el tratamiento de agua residual industrial en reactores UASB a baja temperatura (Kalyuzhnyi et al. 2001, Sato et al. 2006, Tawfik et al. 2008, López y Borzacconi 2009, El-Kamah et al. 2011, Esparza-Soto et al. 2011, 2013).

El objetivo del presente trabajo fue evaluar la eficiencia de remoción (ER) de la demanda química de oxígeno soluble $\left(\mathrm{DQO}_{\mathrm{s}}\right)$, la producción del biogás (PB) y el rendimiento celular experimental $\left(Y_{\exp }\right)$ en un reactor UASB a escala de laboratorio durante el tratamiento de agua residual industrial con dos $\mathrm{CO}$ $\mathrm{V}_{\text {apl }} \mathrm{y}$ temperatura psicrofílica en la ciudad de Toluca, Estado de México.

\section{MATERIALES Y MÉTODOS}

El reactor UASB se construyó a escala de laboratorio con acrílico trasparente de un espesor de $0.8 \mathrm{~cm}$ $\mathrm{y}$ un volumen de $4.5 \mathrm{~L}(10 \times 10 \times 45.5 \mathrm{~cm})$. La parte inferior interna del reactor fue cónica para tener una distribución uniforme del influente. En la salida del reactor se instaló un sedimentador de lamelas para ayudar a la retención de sólidos suspendidos dentro del reactor. El reactor fue alimentado con una bomba peristáltica (marca Cole Palmer, modelo Masterflex, EUA) para mantener el flujo constante y continuo durante las dos etapas experimentales (Fig. 1).

El reactor UASB a escala de laboratorio de esta investigación se inoculó con lodo anaerobio procedente del sedimentador secundario de un reactor UASB piloto, que trato el mismo tipo de agua residual y operó a un rango de temperatura similar a la empleada en esta investigación $\left(18 \pm 0.6^{\circ} \mathrm{C}\right)$ (Esparza-Soto et al. 2013).

El agua residual con la que se alimentó el reactor a escala de laboratorio procedió de una industria chocolatera (Cuadro I). El reactor UASB a escala de laboratorio se operó con dos $\mathrm{COV}_{\text {apl }}$, que se identificaron como etapa experimental I $(1.8 \pm$ $\left.0.8 \mathrm{~kg} \mathrm{DQO} / \mathrm{m}^{3} / \mathrm{d}\right)$ y II $(3.5 \pm 0.9 \mathrm{~kg} \mathrm{DQO} / \mathrm{m} / \mathrm{d})$.

El reactor operó con un TRH de $6 \mathrm{~h}$ y a un rango de temperatura de 18 a $21^{\circ} \mathrm{C}$. Las $\mathrm{COV}_{\text {apl }}$ se obtuvieron diluyendo el agua residual industrial cruda con agua potable, y tuvieron fluctuaciones debidas a variaciones en el flujo y a la degradación de la $\mathrm{DQO}_{\mathrm{s}}$ del influente con el paso del tiempo. Las etapas experimentales I y II duraron 83 y 110 días, respectivamente.

Los parámetros físicos y químicos medidos y analizados fueron $\mathrm{pH}$, temperatura, alcalinidad, $\mathrm{DQO}_{\mathrm{s}}$, ácidos grasos volátiles (AGV), sólidos suspendidos totales (SST) y volátiles (SSV). Los métodos analíticos empleados para la medición y el análisis de los parámetros físicos y químicos se muestran en el cuadro II.

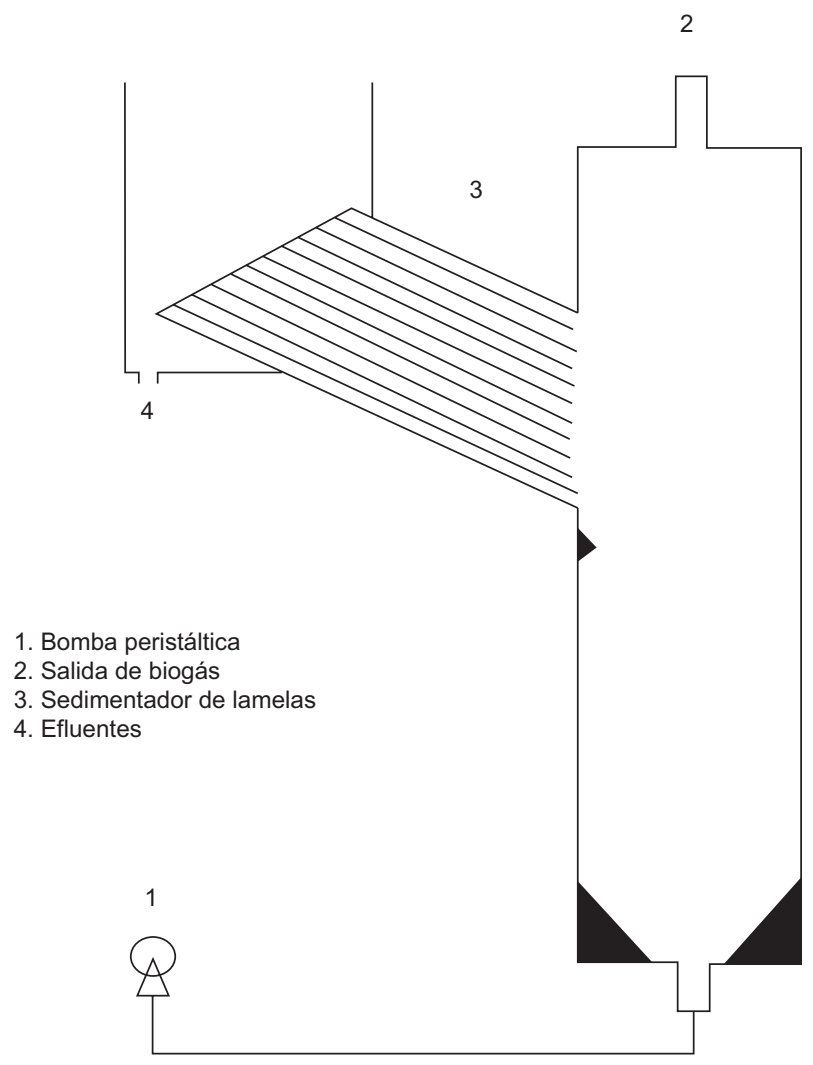

Fig. 1. Diagrama del reactor anaerobio de flujo ascendente rectangular a escala de laboratorio, con volumen total de $4.5 \mathrm{~L}$

CUADRO I. CARACTERIZACIÓN DEL AGUA RESIDUAL INDUSTRIAL CRUDA

\begin{tabular}{|c|c|}
\hline Parámetro & Valor \pm DER \\
\hline $\mathrm{pH}$ & $4.6 \pm 0.8$ \\
\hline Temperatura $\left({ }^{\circ} \mathrm{C}\right)$ & $22.0 \pm 0.1$ \\
\hline $\mathrm{DQO}_{\mathrm{T}}(\mathrm{mg} / \mathrm{L})$ & $6470.0 \pm 537$ \\
\hline $\mathrm{DQO}_{\mathrm{s}}(\mathrm{mg} / \mathrm{L})$ & $4660.0 \pm 367.7$ \\
\hline Sólidos suspendidos totales $(\mathrm{mg} / \mathrm{L})$ & $785.6 \pm 62.1$ \\
\hline Sólidos suspendidos volátiles $(\mathrm{mg} / \mathrm{L})$ & $750.6 \pm 53.8$ \\
\hline Ácidos grasos volátiles (mg ácido acético/L) & $313.8 \pm 13.6$ \\
\hline Alcalinidad $\left(\mathrm{mg} \mathrm{CaCO}_{3} / \mathrm{L}\right)$ & $679.8 \pm 95.9$ \\
\hline $\mathrm{N}-\mathrm{NH}_{3}(\mathrm{mg} / \mathrm{L})$ & $11.0 \pm 1.3$ \\
\hline$P_{\text {total }}(\mathrm{mg} / \mathrm{L})$ & $268.1 \pm 59$ \\
\hline
\end{tabular}

$\mathrm{DQO}_{\mathrm{T}}$ : demanda química de oxígeno total, $\mathrm{DQO}_{\mathrm{s}}$ : demanda química de oxígeno soluble, $\mathrm{N}-\mathrm{NH}_{3}$ : nitrógeno amoniacal, $\mathrm{P}_{\text {Total }}$ : fósforo total, DER: desviación estándar relativa

Los parámetros de control $(\mathrm{pH}$, temperatura y flujo) se midieron por triplicado seis días por semana durante toda la experimentación. Los parámetros de control se midieron para regular la operación del reactor. El pH debe mantenerse cerca de la neutralidad 
CUADRO II. PARÁMETROS FÍSICOS Y QUÍMICOS Y MÉTODOS ANALÍTICOS UTILIZADOS DURANTE LAS ETAPAS EXPERIMENTALES

\begin{tabular}{lll}
\hline Parámetros & Método analítico & Material y equipo \\
\hline $\mathrm{pH}$ & NMX-AA-008-SCFI-2016 (SE 2016) & $\begin{array}{l}\text { Medidor multiparamétrico } \\
\text { (marca YSI, modelo 63) }\end{array}$ \\
\hline Temperatura & NMX-AA-007-SCFI-2013 (SECOFI 2014) & Bureta de 50 mL \\
\hline Alcalinidad & NMX-AA-036-SCFI-2001 (SE 2001a) & $\begin{array}{l}\text { Digestor (marca HACH, modelo DRB 200) Espectrofo- } \\
\text { tómetro (marca Hach, modelo DR 5000) }\end{array}$ \\
\hline DQO $_{\mathrm{T}}$ y DQO & $\begin{array}{l}\text { Método 8000, Método de digestión de reactor } \\
\text { (Hach 2000). }\end{array}$ & $\begin{array}{l}\text { Estufa (marca Lab-Line, modelo Imperial V) y mufla } \\
\text { (marca Barnstead, modelo F6010) }\end{array}$ \\
\hline SST y SSV & NMX-AA-034-SCFI-2001(SE 2001b) & Parrilla eléctrica, material de destilación y titulación \\
\hline AGV & Método de destilación (APHA 2012) &
\end{tabular}

DQO $\mathrm{T}$ : demanda química de oxígeno total, $\mathrm{DQO}_{\mathrm{s}}$ : demanda química de oxígeno soluble, $\mathrm{SST}$ : sólidos suspendidos totales, SSV: sólidos suspendidos volátiles, AGV: ácidos grasos volátiles

debido a que el nivel de $\mathrm{pH}$ óptimo de la digestión anaerobia es de 6.3 a 8.5 (O'Flaherty et al. 2006). La temperatura se midió para conocer en qué rango se operó el reactor y el flujo para mantener el TRH deseado en la presente investigación.

La DQO, SST y SSV se analizaron tres veces por semana. La DQO se analizó para determinar su eficiencia de remoción dentro del reactor. La alcalinidad y los AGV se cuantificaron una vez por semana. Los AGV, SST y SSV se determinaron solamente en el efluente para monitorear una posible acidificación del reactor y calcular el TRC, respectivamente. Los SSV dentro del reactor se determinaron al inicio y final de las etapas experimentales (excepto al inicio de la etapa experimental I) por sextuplicado.

La PB se cuantificó por el método de desplazamiento de líquido (Álvarez et al. 2006, Esparza-Soto et al. 2013) y los resultados obtenidos se corrigieron para condiciones estándar de presión y temperatura (1 atm, $273 \mathrm{~K}\left[0{ }^{\circ} \mathrm{C}\right]$ ) (ecuación 1).

$P B_{S T D}=P B \cdot \frac{P_{o p}}{p} \cdot \frac{T}{T+T_{o p}}$

donde $P B_{\mathrm{STD}}$ es la producción de biogás en condiciones estándar $(\mathrm{L} / \mathrm{d}), P B$ la producción de biogás medido $(\mathrm{L} / \mathrm{d}), P_{\text {op }}$ la presión atmosférica de operación $(0.74 \mathrm{~atm}), P$ la presión estándar $(1 \mathrm{~atm}), T$ la temperatura estándar $(273 \mathrm{~K})$ y $T_{\mathrm{op}}$ la temperatura de operación $(\mathrm{K})$.

La producción de biogás teórica (PBT) se determinó para obtener una aproximación de la cantidad de biogás esperada (ecuación 2). A partir de la ecuación de la ley de los gases ideales se calculó la PBT (Esparza et al. 2013, Tchobanoglous et al. 2014).

$$
\begin{aligned}
P B T= & \frac{n R T_{o p}}{P_{o p}} \times \frac{1 \mathrm{~mol} \mathrm{CH}_{4}}{64 \mathrm{~g} D Q O_{r e m}} \times \\
& \frac{1}{\% \mathrm{de} \mathrm{CH}_{4}} \times(Q \times(S o-S)) \times \\
& \left(1-\frac{1.42 \times Y_{\text {exp }}}{1+b_{H} \times T R C}\right)
\end{aligned}
$$

donde $n$ es el número de moles del gas $\left(1 \mathrm{~mol} \mathrm{CH}_{4}\right)$, $R$ la constante universal de los gases ideales $(0.082057$ $\operatorname{atm~L/mol~K),~} T_{\text {op }}$ la temperatura de operación $(\mathrm{K}), P_{\text {op }}$ la presión atmosférica de operación $(0.74 \mathrm{~atm}), Q$ el flujo (L/d), So la $D Q O_{\text {s }}$ del influente (mg/L), $S$ la $D Q O_{\text {s }}$ del efluente $(\mathrm{mg} / \mathrm{L}), Y_{\text {exp }}$ el rendimiento celular experimental $\left(0.11 \mathrm{mg} \mathrm{SSV} / \mathrm{mg} D Q O_{\text {rem, valor obtenido en }}\right.$ esta investigación), $b_{\mathrm{H}}$ el coeficiente de decaimiento endógeno (0.03 1/d, Tchobanoglous et al. 2014) y TRC el tiempo de retención celular (81 y 95 días para las etapas experimentales I y II, respectivamente).

Los SST y SSV dentro del reactor y del efluente se utilizaron para calcular el TRC al final de cada etapa experimental con la ecuación 3 (Tchobanoglous et al. 2014):

$T R C=\frac{V_{r} \cdot X_{r}}{Q \cdot X_{e}}$

donde $V_{\mathrm{r}}$ es el volumen del reactor (L), $X_{\mathrm{r}}$ la biomasa del reactor (mg SSV/L), Q el flujo (L/d), y $X_{\mathrm{e}}$ la biomasa del efluente (mg SSV/L).

El rendimiento celular experimental $\left(Y_{\exp }\right)$ se calculó para el periodo de tiempo comprendido entre dos cuantificaciones consecutivas de SSV del reactor con la ecuación 4 (Esparza-Soto et al. 2013): 
$Y_{\text {exp }}=\frac{\Delta X_{r}+\Delta X_{e}}{\Delta D Q O_{\text {rem }}}$

donde $\Delta X_{\mathrm{r}}$ es la masa total del lodo acumulado en el interior del reactor durante el periodo de tiempo entre dos cuantificaciones de biomasa del reactor (kg SSV), $\Delta X_{\mathrm{e}}$ la masa total del lodo perdido en el efluente durante el periodo de tiempo entre dos cuantificaciones de $X_{\mathrm{r}}(\mathrm{kg} \mathrm{SSV})$ y $\triangle D Q O_{\text {rem }}$ la masa total del $D Q O_{\mathrm{s}}$ removida $\left(\mathrm{kg} D Q O_{\mathrm{s}}\right.$ rem) durante el periodo de tiempo entre dos cuantificaciones de $X_{\mathrm{r}}$.

\section{RESULTADOS Y DISCUSIÓN}

Los resultados de los parámetros analizados en el reactor UASB se presentan en el cuadro III. El flujo se mantuvo entre $18.1 \pm 3.4$ y $18.6 \pm 2.6 \mathrm{~L} / \mathrm{d}$ durante las dos etapas experimentales. El control del flujo permitió mantener el TRH deseado en el presente trabajo.

El pH del efluente estuvo cercano al neutro en las dos etapas experimentales, mientras que los AGV fueron más bajos (de 31.3 \pm 4.1 a $189.1 \pm 20.9$ mg áci-

CUADRO III. CONDICIONES DE OPERACIÓN Y PARÁMETROS MEDIDOS Y ANALIZADOS EN EL REACTOR ANAEROBIO DE FLUJO ASCENDENTE

\begin{tabular}{|c|c|c|c|}
\hline \multirow[t]{2}{*}{ Parámetro } & \multicolumn{2}{|c|}{ Promedio \pm DER } & \multirow[t]{2}{*}{$\mathrm{n}$} \\
\hline & Influente & Efluente & \\
\hline & \multicolumn{3}{|c|}{ Etapa I } \\
\hline $\mathrm{pH}$ & $7.2 \pm \quad 0.2$ & $7.6 \pm 0.2$ & 73 \\
\hline Temperatura $\left({ }^{\circ} \mathrm{C}\right)$ & $19.2 \pm 1.7$ & $19.0 \pm 1.8$ & 73 \\
\hline $\mathrm{Q}(\mathrm{L} / \mathrm{d})$ & NA & $18.1 \pm 3.4$ & 73 \\
\hline TRH (h) & NA & $6.1 \pm 1.1$ & 73 \\
\hline AGV (mg ácido acético/L) & ND & $31.3 \pm 4.1$ & 2 \\
\hline Alcalinidad $\left(\mathrm{mg} \mathrm{CaCO}_{3} / \mathrm{L}\right)$ & $1134.4 \pm 158.8$ & $1108.9 \pm 82.4$ & 5 \\
\hline $\mathrm{COV}_{\text {apl }}\left(\mathrm{kg} \mathrm{DQO}_{\mathrm{s}} / \mathrm{m}^{3} / \mathrm{d}\right)$ & $1.8 \pm \quad 0.8$ & NA & 19 \\
\hline $\mathrm{COV}_{\text {rem }}\left(\mathrm{kg} \mathrm{DQO} / \mathrm{m}^{3} / \mathrm{d}\right)$ & NA & $1.2 \pm 0.6$ & 19 \\
\hline $\mathrm{DQO}_{\mathrm{s}}(\mathrm{mg} / \mathrm{L})$ & $426.5 \pm 133.8$ & $125.9 \pm 80.6$ & 22 \\
\hline $\mathrm{ER}(\%)$ & NA & 70.4 & 22 \\
\hline $\operatorname{ER}(\%)^{*}$ & NA & $90.3 \pm 1.3$ & 4 \\
\hline Duración del periodo (d) & 83 & NA & NA \\
\hline \multirow[t]{2}{*}{$\mathrm{PB}(\mathrm{L} / \mathrm{d})$} & NA & $1.6 \pm 0.8$ & 51 \\
\hline & \multicolumn{3}{|c|}{ Etapa II } \\
\hline $\mathrm{pH}$ & $7.2 \pm \quad 0.2$ & $7.7 \pm 0.2$ & 92 \\
\hline Temperatura $\left({ }^{\circ} \mathrm{C}\right)$ & $18.2 \pm 2$ & $17.8 \pm 2$ & 92 \\
\hline $\mathrm{Q}(\mathrm{L} / \mathrm{d})$ & NA & $18.6 \pm 2.6$ & 92 \\
\hline TRH (h) & NA & $5.9 \pm 0.8$ & 92 \\
\hline AGV (mg ácido acético/L) & ND & $189.1 \pm 20.9$ & 6 \\
\hline Alcalinidad $\left(\mathrm{mg} \mathrm{CaCO}_{3} / \mathrm{L}\right)$ & $1449.8 \pm 336.3$ & $1603.0 \pm 172$ & 11 \\
\hline $\mathrm{COV}_{\text {apl }}\left(\mathrm{kg} \mathrm{DQO} / \mathrm{m}^{3} / \mathrm{d}\right)$ & $3.5 \pm \quad 0.9$ & NA & 26 \\
\hline $\mathrm{COV}_{\text {rem }}\left(\mathrm{kg} \mathrm{DQO}_{\mathrm{s}} / \mathrm{m}^{3} / \mathrm{d}\right)$ & NA & $2.6 \pm 0.7$ & 26 \\
\hline $\mathrm{DQO}_{\mathrm{s}}(\mathrm{mg} / \mathrm{L})$ & $830.6 \pm 162$ & $198.6 \pm 147$ & 28 \\
\hline $\mathrm{ER}(\%)$ & NA & 77.1 & 28 \\
\hline $\operatorname{ER}(\%)^{*}$ & NA & $90.6 \pm 3.1$ & 13 \\
\hline Duración del periodo (d) & 110 & NA & NA \\
\hline $\mathrm{PB}(\mathrm{L} / \mathrm{d})$ & NA & $3.5 \pm 1.3$ & 68 \\
\hline
\end{tabular}

Q: caudal, TRH: tiempo de retención hidráulico, AGV: ácidos grasos volátiles, $\mathrm{COV}_{\text {apl: }}$ carga orgánica volumétrica aplicada, $\mathrm{COV}_{\text {rem }}$ : carga orgánica volumétrica removida, $\mathrm{DQO}_{\mathrm{s}}$ : demanda química de oxígeno soluble, ER: eficiencia de remoción, PB: producción de biogás, DER: desviación estándar relativa, n: número de muestras, NA: no aplica, ND: no determinado

*En estado estacionario; en la etapa experimental I comprende del día 71 al 83 de operación, y en la etapa experimental II del día 151 al 193 de operación 
CUADRO IV. CONCENTRACIONES DE SÓLIDOS SUSPENDIDOS TOTALES Y SÓLIDOS SUSPENDIDOS VOLÁTILES DEL EFLUENTE Y DENTRO DEL REACTOR (AL FINAL DE LA ETAPA EXPERIMENTAL) Y TIEMPO DE RETENCIÓN CELULAR

\begin{tabular}{lccccccc}
\hline $\left.\begin{array}{l}\text { Etapa/COV } \\
(\mathrm{kg} \mathrm{DQO}\end{array} \mathrm{C}_{\mathrm{s}} / \mathrm{m} / \mathrm{d}\right)$ & \multicolumn{2}{c}{ Efluente } & \multicolumn{2}{c}{$\mathrm{SSV} / \mathrm{SST}$} & \multicolumn{2}{c}{ Reactor } & \multicolumn{2}{c}{ SSV/SST } & TRC \\
$(\mathrm{mg} \mathrm{SST} / \mathrm{L})$ & $(\mathrm{mg} \mathrm{SSV} / \mathrm{L})$ & $(\%)$ & $(\mathrm{mg} \mathrm{SST} / \mathrm{L})$ & $(\mathrm{mg} \mathrm{SSV} / \mathrm{L})$ & $(\%)$ & $(\mathrm{d})$ \\
\hline $\mathrm{I}(1.8 \pm 0.8)$ & $44.1 \pm 21.0$ & $35.6 \pm 18.4$ & 79.9 & 16175 & 11550 & 71.4 & 81 \\
$\mathrm{II}(3.5 \pm 0.9)$ & $56.6 \pm 14.9$ & $45.3 \pm 11.0$ & 80.5 & 33175 & 23900 & 72.0 & 95 \\
\hline
\end{tabular}

$\mathrm{COV}_{\mathrm{apl}}$ : carga orgánica volumétrica aplicada, $\mathrm{DQO}_{\mathrm{s}}$ : demanda química de oxígeno soluble, $\mathrm{SST}$ : sólidos suspendidos totales, SSV: sólidos suspendidos volátiles, TRC: tiempo de retención celular

do acético/L) que la concentración máxima sugerida (250 mg ácido acético/L) (Crites y Tchnobanoglous 2000); la alcalinidad fue alta (de $1109 \pm 82$ a 1603 $\left.\pm 172 \mathrm{mg} \mathrm{CaCO}_{3} / \mathrm{L}\right)$ respecto de la concentración mínima recomendada (1000 $\left.\mathrm{mg} \mathrm{CaCO}_{3} / \mathrm{L}\right)($ Crites y Tchnobanoglous 2000).

El pH del efluente cercano a la neutralidad, los AGV bajos y la alcalinidad alta indicaron que el tratamiento anaerobio se mantuvo estable, lo cual se debió a que la alcalinidad fue suficiente para amortiguar los cambios de $\mathrm{pH}$ causados por la formación de AGV durante la digestión anaerobia (Isik y Sponza 2005, Henze et al. 2008). Por lo tanto, el monitoreo del pH, el control de la alcalinidad y la determinación de los AGV permitieron evitar la acidificación del reactor (Cuadro III).

Los SST y SSV en el efluente fueron bajos durante las dos etapas experimentales de las dos etapas experimentales (Cuadro IV). Los SSV en el efluente durante las etapas experimentales I y II fueron de 56.6 \pm 14.9 y de $45.3 \pm 11.0 \mathrm{mg} / \mathrm{L}$, respectivamente. Los SST en el efluente durante las dos etapas experimentales fueron menores a la concentración recomendada por Foresti et al. (2006) (50 a $100 \mathrm{mg} / \mathrm{L})$.

La baja concentración de SST y SSV en el efluente en el presente trabajo se debió a la presencia de un sedimentador de lamelas en la salida del reactor y a la baja velocidad ascensional $(0.05 \mathrm{~m} / \mathrm{h})$ dentro de éste. Por otro lado, los SST y SSV en el efluente tuvieron concentraciones similares a los reportados por Turkdogan-Aydinol et al. (2011).

La calidad del efluente respecto a la concentración de SST y SSV en el presente trabajo fue satisfactoria y comparable con la literatura (Foresti et al. 2006, Turkdogan-Aydinol et al. 2011). La relación SSV/ SST en el efluente de las dos etapas experimentales fue de 79.9 y $80.5 \%$. Esta alta relación indicó que la mayor parte de los SSV fugados en el efluente provenían del lodo biológico anaerobio presente dentro del reactor.
La concentración de SST y SSV dentro del reactor se incrementó al doble durante la etapa experimental II. La baja concentración de SSV en el efluente favoreció el aumento de la cama de lodo dentro del reactor, y esto a su vez permitió tener un tratamiento anaerobio estable.

La relación SSV/SST dentro del reactor en las etapas experimentales I y II fue de 71.4 y $72.0 \%$, respectivamente. Esta relación indicó que la cama de lodo estuvo conformada mayormente por lodo biológico anaerobio (Tchobanoglous et al. 2014) y no hubo acumulación de sólidos suspendidos inertes dentro del reactor.

El TRC se incrementó cuando la $\mathrm{COV}_{\text {apl }}$ aumentó (Cuadro IV). El TRC de las dos etapas experimentales (81 y 95 días) fue más largo que el mínimo requerido para mantener la actividad metanogénica a baja temperatura (TRC 75 días) (Lettinga et al. 2001). El TRC largo fue provocado por la baja concentración de SSV en el efluente, lo cual incrementó los SSV dentro del reactor. El TRC largo permitió obtener una ER alta (90 \%) durante el estado estacionario de las dos etapas experimentales.

La ER del reactor UASB se evaluó durante las dos etapas experimentales (Fig. 2). La ER durante

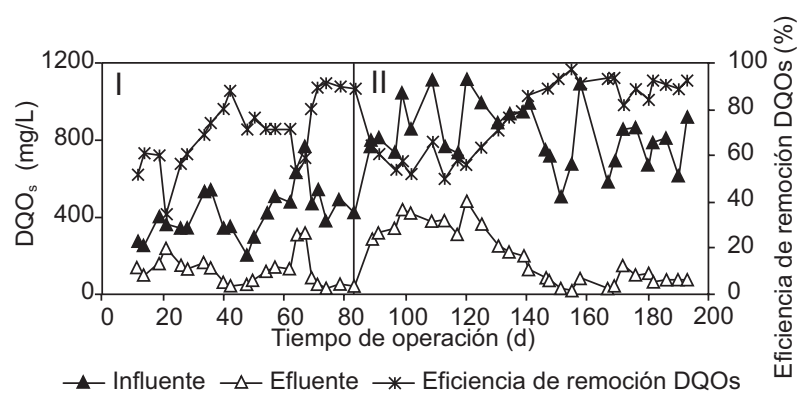

Fig. 2. Eficiencia de remoción de la demanda química de oxígeno $\left(\mathrm{DQO}_{\mathrm{s}}\right)$ del influente y efluente durante 193 días de operación en un reactor anaerobio de flujo ascendente 
toda la etapa experimental I fue variable, mientras que en la etapa experimental II se incrementó con el paso del tiempo de operación del reactor UASB, independientemente de la fluctuación de la $\mathrm{DQO}_{\mathrm{s}}$ del influente.

El estado estacionario de las etapas experimentales I y II se alcanzó a partir de los días 71 y 151 de operación, respectivamente. El reactor UASB tardó en alcanzar el estado estacionario en ambas etapas experimentales debido a la baja concentración inicial de SSV en su interior. En estado estacionario, la ER de las etapas experimentales I y II fue de $90.3 \pm 1.3$ y $90.6 \pm 3.1 \%$, respectivamente.

La ER fue mayor que el valor teórico de van Haandel et al. (2006) (80\%) y que lo reportado en la literatura por Sato et al. (2006) y Tawfik et al. (2008), quienes operaron sus reactores en condiciones similares de $\mathrm{COV}_{\text {apl }}$ y temperatura. La ER del presente trabajo posiblemente fue mayor porque se trató un solo tipo de agua residual industrial, a diferencia de los autores mencionados, quienes usaron una mezcla de diferentes tipos de aguas residuales.

Trabajar con un solo tipo de agua residual industrial evitó posibles variaciones en la calidad del agua, la cual puede influir en el tratamiento anaerobio. Además, el lodo anaerobio del presente trabajo estaba adaptado previamente al mismo tipo de agua residual industrial y a la baja temperatura de operación. Con lo antes descrito se favoreció la ER del presente trabajo.

La correlación lineal entre la $\mathrm{COV}_{\text {apl }}$ y la carga orgánica volumétrica removida $\left(\mathrm{COV}_{\text {rem }}\right)$ de las etapas experimentales I y II se muestran en la figura 3. El alto coeficiente de determinación $\left(\mathrm{R}^{2}=0.7605\right)$ indica que los datos experimentales de $\mathrm{COV}_{\text {apl }}$ y $\mathrm{COV}_{\text {rem }}$ estuvieron relacionados entre sí.

La $\mathrm{COV}_{\text {rem }}$ aumentó cuando la $\mathrm{COV}_{\text {apl }}$ se incrementó. La pendiente de la correlación lineal indicó el porcentaje de ER global de las dos etapas experimentales. La ER global mostrada en la figura 3 (74\%) fue igual a la calculada con la $\mathrm{DQO}_{\mathrm{s}}$ del influente y efluente y cercana al valor teórico de van Haandel et al. (2006) (80\%).

La ER global obtenida indicó que el tratamiento anaerobio para este tipo de agua residual industrial fue eficiente y comparable con ER teóricas para reactores operados a temperaturas mayores de $20{ }^{\circ} \mathrm{C}$ (van Haandel et al. 2006).

$\mathrm{La} \mathrm{PB}$ y la $\mathrm{COV}_{\text {rem }}$ durante ambas etapas experimentales se muestran en la figura 4. En general se observa que la $\mathrm{PB}$ se incrementó conforme la $\mathrm{COV}_{\text {rem }}$ aumentó. Los días 48 y 68 de operación se presentaron dos disminuciones de la PB debido a problemas operativos. La $\mathrm{PB}_{\mathrm{STD}}$ promedio de las etapas expe-

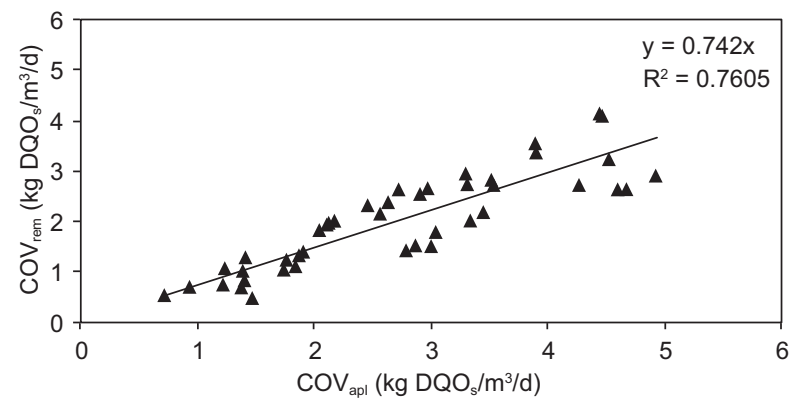

Fig. 3. Correlación lineal entre la carga orgánica volumétrica aplicada $\left(\mathrm{COV}_{\text {apl }}\right)$ y la carga orgánica volumétrica removida $\left(\mathrm{COV}_{\text {rem }}\right)$ del reactor anaerobio de flujo ascendente durante las etapas experimentales I y II

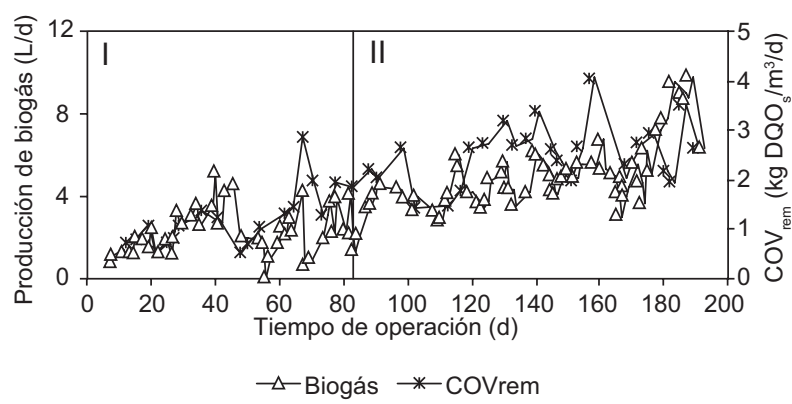

Fig. 4. Producción de biogás y carga orgánica volumétrica removida $\left(\mathrm{COV}_{\text {rem }}\right)$ en las etapas experimentales I y II

rimentales I y II fue de $1.6 \mathrm{~L} / \mathrm{d}\left(0.3 \mathrm{~L}_{\text {biogás }} / \mathrm{L} / \mathrm{d}\right)$ y 3.5 $\mathrm{L} / \mathrm{d}\left(0.8 \mathrm{~L}_{\text {biogás }} / \mathrm{L} / \mathrm{d}\right)$, respectivamente.

En el presente trabajo se obtuvo mayor $\mathrm{PB}_{\mathrm{STD}}$ que Sponza y Uluköy (2008), quienes trabajaron con la misma $\mathrm{COV}_{\text {apl }}$ de la etapa experimental II $(0.3$ $\mathrm{L}_{\text {biogás }} / \mathrm{L} / \mathrm{d}$ a $3.5 \pm 0.9 \mathrm{~kg} \mathrm{DQO} / \mathrm{m}^{3} / \mathrm{d}$ ) y una temperatura de operación mayor $\left(37^{\circ} \mathrm{C}\right)$. La ER y el origen alimenticio del agua residual pudieron favorecer a la $\mathrm{PB}_{\text {STD }}$ del presente trabajo a diferencia de lo reportado por Sponza y Uluköy (2008), quienes utilizaron agua residual sintética con un contaminante.

La correlación lineal entre la $\mathrm{PB}$ y la $\mathrm{COV}_{\text {rem }}$ muestra que la $\mathrm{PB}$ se incrementó cuando la $\mathrm{COV}_{\text {rem }}$ aumentó (Fig. 5). La pendiente de esta correlación indicó que se produjeron $1.79 \mathrm{~L}$ de biogás por cada $\mathrm{kg} \mathrm{DQO}_{\text {s removida }}\left(\mathrm{DQO}_{\mathrm{s} \mathrm{rem}} / \mathrm{m}^{3} \mathrm{~d}\right)\left(\mathrm{R}^{2}=0.6566\right)$. A partir de la pendiente de la correlación lineal entre la $\mathrm{COV}_{\text {rem }}$ y la PB se calculó el rendimiento de metano.

La pendiente de la correlación lineal se dividió entre el volumen del reactor y se consideró que el $84 \%$ del biogás fue metano (valor determinado por Ávila-Arias [2015] para el mismo lodo anaerobio, 


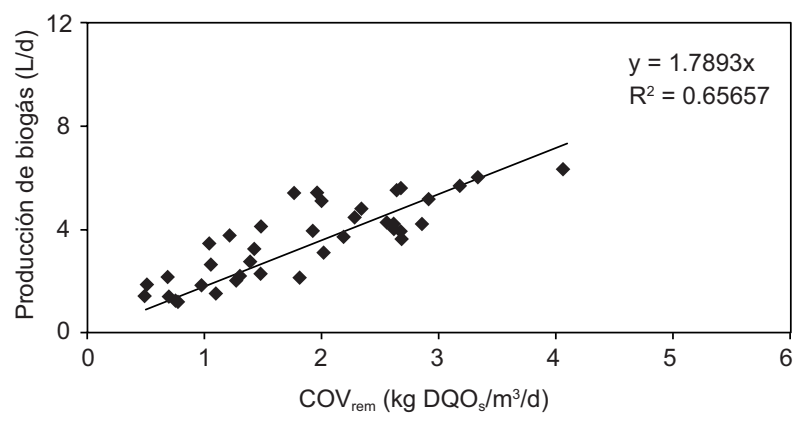

Fig. 5. Correlación entre la carga orgánica volumétrica $\left(\mathrm{COV}_{\text {rem }}\right)$ y la producción de biogás durante la operación del reactor anaerobio de flujo ascendente

el mismo tipo de agua residual y temperatura de operación). El rendimiento del metano fue de 231.6 $\mathrm{L} \mathrm{CH}_{4} / \mathrm{kg} \mathrm{DQO}_{\text {s rem }}\left(1 \mathrm{~atm}\right.$ y $\left.0{ }^{\circ} \mathrm{C}\right)$, siendo este valor menor al teórico (350 $\mathrm{L} \mathrm{CH}_{4} / \mathrm{kg} \mathrm{DQO}_{\text {s rem }}$ ) (Henze et al. 2008) y mayor a los reportados en la literatura de $167 \mathrm{~L} \mathrm{CH}_{4} / \mathrm{kg} \mathrm{DQO}$ s rem (Singh y Viraraghavan 2004) y $174 \mathrm{~L} \mathrm{CH}_{4} / \mathrm{kg} \mathrm{DQO}_{\text {s rem }}$ (Esparza-Soto et al. 2013).

La diferencia entre los valores de rendimiento del metano del presente trabajo y la literatura puede atribuirse a la composición del metano en el biogás, que puede variar dependiendo del tipo y concentración de materia orgánica presente en el agua residual (Noyola et al. 2006).

La correlación lineal entre la PB y la PBT se observa en la figura 6. La pendiente de la correlación lineal indicó el porcentaje de biogás teórico recuperado en el reactor UASB. En la presente investigación se recuperó el $68 \%$ de la PBT $\left(\mathrm{R}^{2}\right.$ $=0.7075)$. Este bajo porcentaje de recuperación puede atribuirse principalmente a pérdidas de metano disuelto en el efluente ocasionadas por la baja temperatura de operación, ya que la solubilidad de un gas en un fluido aumenta cuando la temperatura disminuye (Brown et al. 2009). Sin embargo, el

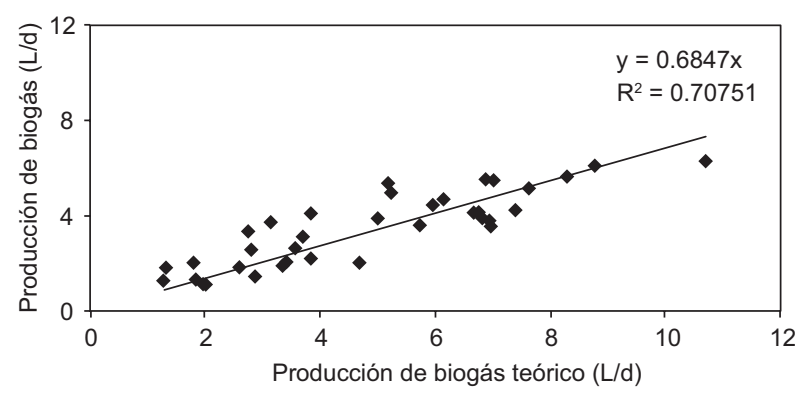

Fig. 6. Correlación lineal entre la producción de biogás teórico y la producción de biogás durante las etapas experimentales I y II porcentaje de biogás recuperado fue cercano al $65 \%$ reportado por Ávila-Arias (2015), al $74 \%$ reportado por Lara-Domínguez (2013) con las mismas condiciones de operación que las del presente trabajo y al $71 \%$ informado por Bandara et al. (2011) con agua residual sintética a $15^{\circ} \mathrm{C}$.

El $Y_{\exp }$ calculado para la etapa de experimentación II fue de $0.11 \mathrm{~kg} \mathrm{SSV} / \mathrm{kg} \mathrm{DQO}$ s rem a $17.8^{\circ} \mathrm{C}$. El $Y_{\exp }$ fue más alto que el valor clásico reportado por Tchobanoglous et al. (2014) (0.08 kg SSV/kg DQO). El $Y_{\text {exp }}$ alto del presente estudio pudo deberse a que la tasa de crecimiento de los microorganismos anaerobios se incrementó en condiciones psicrofílicas, tal como fue reportado por van Lier et al. (1997).

El incremento de los SSV dentro del reactor durante la etapa experimental II (11 550 a 23900 $\mathrm{mg} \mathrm{SSV/L)} \mathrm{y} \mathrm{la} \mathrm{baja} \mathrm{concentración} \mathrm{de} \mathrm{SSV} \mathrm{en} \mathrm{el}$ efluente $(45.3 \pm 11.0 \mathrm{mg} \mathrm{SSV} / \mathrm{L})$ indicaron una alta tasa de formación de microorganismos anaerobios, la cual es controlada por el $Y_{\text {exp. }}$ La literatura reporta valores elevados similares de $Y_{\exp }$ de $0.18 \mathrm{~kg} \mathrm{SSV} /$ $\mathrm{kg}$ DQO a $20^{\circ} \mathrm{C}$ (Singh y Viraraghavan 2002) y de $0.2 \pm 0.04 \mathrm{~kg} \mathrm{SSV} / \mathrm{kg}$ DQO a $18^{\circ} \mathrm{C}$ (Esparza-Soto et al. 2013).

\section{CONCLUSIONES}

El pH del efluente cercano al neutro, los AGV bajos y la alcalinidad alta permitieron obtener un tratamiento anaerobio estable porque los microorganismos estuvieron en equilibrio entre sí, evitando la acidificación del reactor.

La baja concentración de SST y SSV del efluente favoreció la acumulación de SSV en la cama de lodo anaerobio. Lo anterior benefició la estabilidad de la ER durante la operación del reactor UASB.

El reactor UASB trató eficientemente el agua residual industrial con $\mathrm{COV}_{\text {apl }}$ intermedia $(1.8 \pm 0.8$ a $\left.3.5 \pm 0.9 \mathrm{~kg} \mathrm{DQO} / \mathrm{m}^{3} / \mathrm{d}\right)$ y un TRH corto $(6 \mathrm{~h})$ a temperatura psicrofílica de operación $\left(18-19^{\circ} \mathrm{C}\right)$.

El TRC largo y el lodo anaerobio adaptado previamente a la temperatura y al tipo de agua residual industrial, permitieron alcanzar ER altas en el estado estacionario $(90.3 \pm 1.3 \%$ y $90.6 \pm$ $3.1 \%$ durante las etapas experimentales I y II, respectivamente).

La PB se incrementó cuando la $\mathrm{COV}_{\text {rem }}$ aumentó.

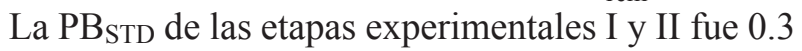
y $0.8 \mathrm{~L}_{\text {biogás }} / \mathrm{L} / \mathrm{d}$, respectivamente.

El bajo porcentaje de biogás recuperado $(68 \%)$ en el presente trabajo se debió a que la solubilidad del metano en el efluente aumentó a temperatura baja. 
El $Y_{\text {exp }}$ fue alto comparado con el valor de Tchobanoglous et al. (2014), lo cual puede atribuirse a la influencia de la baja temperatura de operación sobre la tasa de crecimiento de los microorganismos anaerobios.

\section{AGRADECIMIENTOS}

Los autores agradecen al CONACYT (Proyecto núm. 182696) por el apoyo económico para el estudio.

\section{REFERENCIAS}

Akila G. y Chandra T.S. (2007). Performance of an UASB reactor treating synthetic wastewater at low-temperature using cold-adapted seed slurry. Process Biochem. 42 (3), 466-471. DOI: 10.1016/j.procbio.2006.09.010

Álvarez J.A., Ruiz I., Gómez M., Presas J. y Soto M. (2006). Start-up alternatives and performance of an UASB pilot plant treating diluted municipal wastewater at low temperature. Bioresource Technol. 97 (14), 1640-1649. DOI: 10.1016/j.biortech.2005.07.033

APHA (2012). Standard methods for the examination of water and wastewater. 22a ed. American Public Health Association, Washington, EUA, 1325 pp.

Atuanya E.I. y Chakrabarti T. (2004). Kinetics of biotransformation of 2, 4-dichlorophenol using UASB-reactor. Environ. Monit. Assess. 96 (1-3), 129-141. DOI: 10.1023/B:EMAS.0000031720.86980.2e

Ávila-Árias J.A. (2015). Cuantificación de la actividad metanogénica específica y de la producción de metano en un reactor anaerobio operado a baja temperatura. Tesis de Doctorado. Centro Interamericano de Recursos del Agua-Facultad de Ingeniería, Universidad Autónoma del Estado de México, México, 140 pp.

Bandara W.M., Satoh H., Sasakawa H., Nakahara Y., Takahashi M. y Okabe S. (2011). Removal of residual dissolved methane gas in an upflow anaerobic sludge blanket reactor treating low-strength wastewater at low temperature with degassing membrane. Water Res. 45 (11), 3533-3540. DOI: 10.1016/j.watres.2011.04.030

Bandara W.M., Kindaichi T., Satoh H., Sasakawa M., Nakahara Y., Takahashi M. y Okabe S. (2012). Anaerobic treatment of municipal wastewater at ambient temperature: Analysis of archaeal community structure and recovery of dissolved methane. Water Res. 46 (17), 5756-5764. DOI: 10.1016/j.watres.2012.07.061

Brown T.L., LeMay H.E., Burstin B.E. y Murphy C.J. (2009). Química: la ciencia central. 11a ed. Pearson Educación, México, 1244 pp.
Chou H.-H., Huang J.-S. y Hong W.F. (2004). Temperature dependency of granule characteristics and kinetic behavior in UASB reactors. J. Chem. Technol. Biot. 79, 797-808.

DOI: $10.1002 /$ jctb.999

Crites R. y Tchobanoglous G. (2000). Tratamiento de aguas residuales en pequeñas poblaciones. McGraw-Hill, Santa Fe de Bogotá, Colombia, 776 pp.

Diamantis V.I. y Aivasidis A. (2007). Comparison of single- and two-stage UASB reactors used for anaerobic treatment of synthetic fruit wastewater. Enzyme Microb. Tech. 42 (1), 6-10.

DOI: 10.1016/j.enzmictec.2007.07.014

Donoso-Bravo A., Bandara W.M.K.R.T.W., Satoh H. y Ruiz-Filippi G. (2013). Explicit temperature-based model for anaerobic digestion: Application in domestic wastewater treatment in a UASB reactor. Bioresource Technol. 133, 437-442.

DOI: $10.1016 /$ j.biortech.2013.01.174

El-Kamah H., Mahmoud M. y Tawfik A. (2011). Performance of down-flow hanging sponge (DHS) reactor coupled with up-flow anaerobic sludge blanket (UASB) reactor for treatment of onion dehydration wastewater. Bioresource Technol. 102 (14), 70297035. DOI: 10.1016/j.biortech.2011.04.017

El-Sheikh M.A., Saleh H.I., Flora J.R. y AbdEl-Ghany M.R. (2011). Biological tannery wastewater treatment using two stage UASB reactors. Desalination 276 (13), 253-259.

DOI: $10.1016 / j$.desal.2011.03.060

Esparza-Soto M., Solís-Morelos C. y Hernández-Torres J.J. (2011). Anaerobic treatment of a medium strength industrial wastewater at low-temperature and short hydraulic retention time: A pilot-scale experience. Water Sci. Technol. 64 (8), 1629-1635.

DOI: $10.2166 /$ wst.2011.741

Esparza-Soto M., Arzate-Archundia O., Solis-Morelos C. y Fall C. (2013). Treatment of a chocolate industry wastewater in a pilot-scale low-temperature UASB reactor operated at short hydraulic and sludge retention time. Water Sci. Technol. 67 (6), 1353-1361.

DOI: 10.2166/wst.2013.010

Farajzadehha S., Mirbagheria S.A., Farajzadehha Soroush y Shayegan J. (2012). Lab scale study of HRT and OLR optimization in UASB reactor for pretreating fortified wastewater in various operational temperatures. APCBEE Proc. 1, 90-95.

DOI: $10.1016 /$ j.apcbee.2012.03.016

Foresti E., Zaiat M. y Vallero M. (2006). Anaerobic processes as the core technology for sustainable domestic wastewater treatment: Consolidated applications, new trends, perspectives, and challenges. Rev. Environ. Sci. Bio. 5, 3-19. DOI: 10.1007/s11157-005-4630-9 
Grady Jr L., Daigger G.T. y Lim H.C. (1999). Biological wastewater treatment. 2a ed. Marcel Dekker, Nueva York, EUA, 1076 pp.

Hach (2000). Manual de análisis de agua. Hach company. 3da ed. Loveland, Colorado, EUA, 220 pp.

Henze M., van Loosdrecht M.C.M., Ekama G.A. y Brdjanovic D. (2008). Biological wastewater treatment. Principles, modelling and design. IWA publishing, Londres, Reino Unido, 528 pp.

Hinken L., Huber M., Weichgrebe D. y Rosenwinkel K.H. (2014). Modified ADM1 for modelling an UASB reactor laboratory plant treating starch wastewater and synthetic substrate load tests. Water Res. 64, 82-92. DOI: $10.1016 /$ j.watres.2014.06.044

Isik M. y Sponza D.T. (2005). Effects of alkalinity and cosubstrate on the performance of an upflow anaerobic sludge blanket (UASB) reactor through decolorization of Congo Red azo dye. Bioresource Technol. 96 (5), 633-643. DOI: 10.1016/j.biortech.2004.06.004

Kalyuzhnyi S.V., Gladchenko M.A., Sklyar V.I., Kizimenko Y.S. y Shcherbakov S.S. (2001). One- and two-stage upflow anaerobic sludge-bed reactor pretreatment of winery wastewater at $4-10^{\circ} \mathrm{C}$. Appl. Biochem. Biotech. 90 (2), 107-124.

Lara-Domínguez F. (2013). Recuperación y utilización del biogás producido por un reactor UASB para el incremento de su desempeño. Tesis de Maestría. Centro Interamericano Recursos del Agua-Facultad de Ingeniería, Universidad Autónoma del Estado de México, México, 92 pp.

Lettinga G., Rebac S. y Zeeman G. (2001). Challenge of psychrophilic anaerobic wastewater treatment. Trends Biotechnol. 19 (9), 363-370.

DOI: 10.1016/S0167-7799(01)01701-2

Li W., Chen H., Jin Y., Zhang H., Niu Q., Qi W., Zhang Y., Li Y. y Gao Y. (2015a). Treatment of 3,4,5 trimethoxybenzaldehyde and di-bromo-aldehyde manufacturing wastewater by the coupled fenton pretreatment and UASB reactor with emphasis on optimization and chemicals analysis. Sep. Purif. Technol. 142, 40-47. DOI: 10.1016/j.seppur.2014.12.013

Li W., Niu Q., Zhang H., Tian Z., Zhang Y., Gao Y., Li Y., Nishimura O. y Yang M. (2015b). UASB treatment of chemical synthesis-based pharmaceutical wastewater containing rich organic sulfur compounds and sulfate and associated microbial characteristics. Chem. Eng. J. 260, 55-63. DOI: 10.1016/j.cej.2014.08.085

López I. y Borzacconi L. (2009). Modelling a full scale UASB reactor using a COD global balance approach and state observers. Chem. Eng. J. 146 (1), 1-5. DOI: $10.1016 /$ j.cej.2008.05.007

Lu X., Zhen G., Ledezma-Estrada A., Chen M., Ni J., Hojo T., Kubota K. y Li Y. (2015). Operation performance and granule characterization of upflow anaerobic sludge blanket (UASB) reactor treating wastewater with starch as the sole carbon source. Bioresource Technol. 180, 264-273.

DOI: 10.1016/j.biortech.2015.01.010

Mahmoud N., Zeeman G., Gijzenb H. y Lettinga G. (2004). Anaerobic sewage treatment in a one-stage UASB reactor and a combined UASB-digester system. Water Res. 38 (9), 2348-2358.

DOI: $10.1016 /$ j.watres.2004.01.041

Nacheva P.M., Chávez G.M., Chacón J.M. y Chil A.C. (2009). Treatment of cane sugar mill wastewater in an upflow anaerobic sludge bed reactor. Water Sci. Technol. 60 (5), 1347-1352. DOI: 10.2166/wst.2009.402

Noyola A., Morgan-Sagastume J.M. y López-Hernández J.E. (2006). Treatment of biogas produced in anaerobic reactors for domestic wastewater: Odor control and energy/resource recovery. Environ. Sci. Bio. 5 (1), 93-114. DOI: 10.1007/s11157-005-2754-6

O'Flaherty V., Collins G. y Mahony T. (2006). The microbiology and biochemistry of anaerobic bioreactors with relevance to domestic sewage treatment. Rev. Environ. Sci. Bio. 5, 39-55. DOI: 10.1007/s11157-005-5478-8

Parawira W., Murto M., Zvauya R. y Mattiasson B. (2006). Comparative performance of a UASB reactor and an anaerobic packed-bed reactor when treating potato waste leachate. Renew. Energ. 31 (6), 893-903.

DOI: 10.1016/j.renene.2005.05.013

Qiu G., Song Y., Zeng P., Duan L. y Xiao S. (2013). Combination of upflow anaerobic sludge blanket (UASB) and membrane bioreactor (MBR) for berberine reduction from wastewater and the effects of berberine on bacterial community dynamics. J. Hazard. Mater. 246-247, 34-43. DOI: 10.1016/j.jhazmat.2012.12.010

Rizvi H., Ahmad N., Abbas F., Bukhari I.F., Yasar A., Ali S., Yasmeen R. y Riaz M. (2015). Start-up of UASB reactors treating municipal wastewater and effect of temperature/sludge age and hydraulic retention time (HRT) on its performance. Arabian J. Chem. 8 (6), 780-786. DOI: 10.1016/j.arabjc.2013.12.016

Saha S., Badhe N., Vrieze J.D., Biswas R. y Nandy T. (2015). Methanol induces low temperature resilient methanogens and improves methane generation from domestic wastewater at low to moderate temperatures. Bioresource Technol. 189, 370-378.

DOI: 10.1016/j.biortech.2015.04.056

Sato N., Okubo T., Onodera T., Ohashi A. y Harada H. (2006). Prospects for a self-sustainable sewage treatment system: A case study on full-scale UASB system in India's Yamuna River Basin. J. Environ. Manage. 80 (3), 198-207. DOI: 10.1016/j.jenvman.2005.08.025

SE (2016). Norma Oficial Mexicana NMX-AA-008-SCFI-2016. Análisis de agua-medición de $\mathrm{pH}$ en aguas 
naturales, residuales y residuales tratadas-método de prueba. Secretaria de Economía, Diario Oficial de la Federación, 9 de septiembre.

SE (2001a). Norma Oficial Mexicana NMX-AA-036-SCFI-2001. Análisis de agua. Determinación de acidez y alcalinidad en aguas naturales, residuales y residuales tratadas. Método de prueba. Secretaría de Economía, Diario Oficial de la Federación, 1 de agosto.

SE (2001b). Norma Oficial Mexicana NMX-AA034-SCFI-2001. Análisis de agua. Determinación de sólidos y sales disueltas en aguas naturales, residuales y residuales tratadas. Método de prueba. Secretaría de Economía, Diario Oficial de la Federación, 1 de agosto.

SECOFI (2014). Norma Oficial Mexicana NMX-AA007-SCFI-2013. Análisis de agua. Determinación de la temperatura en aguas naturales, residuales y residuales tratadas. Método de prueba. Secretaria de Comercio y Fomento Industrial, Diario Oficial de la Federación, 23 de enero.

Senthilkumar M., Gnanapragasam G., Arutchelvan V. y Nagarajan S. (2011). Treatment of textile dyeing wastewater using two-phase pilot plant UASB reactor with sago wastewater as co-substrate. Chem. Eng. J. 166 (1), 10-14. DOI: 10.1016/j.cej.2010.07.057

Singh K.S. y Viraraghavan T. (2002). Effect of temperature on bio-kinetic coefficients in UASB treatment of municipal wastewater. Water Air Soil Poll. 136 (1-4), 243-254.

Singh K.S. y Viraraghavan T. (2004). Municipal wastewater treatment by UASB process: Start-up at $20{ }^{\circ} \mathrm{C}$ and operation at low temperatures. Environ. Technol. 25 (6), 621-634. DOI: 10.1080/09593330.2004.9619352

Sponza D.T. y Uluköy A. (2008). Kinetic of carbonaceous substrate in an upflow anaerobic sludge blanket (UASB) reactor treating 2,4 dichlorophenol (2,4 DCP). J. Environ. Manage. 86 (1), 121-131. DOI: 10.1016/j.jenvman.2006.11.030

Tawfik A., Sobheyb M. y Badawy M. (2008). Treatment of a combined dairy and domestic wastewater in an up-flow anaerobic sludge blanket (UASB) reactor followed by activated sludge (AS system). Desalination 227 (1-3), 167-177. DOI: 10.1016/j.desal.2007.06.023
Tchobanoglous G., Stensel H. D., Tsuchihashi R., Burton F. L. Abu-Orf M., Bowden G. y Pfrang W. (2014). Wastewater engineering: Treatment and resource recovery. 5a ed. McGraw-Hill, Nueva York, EUA, 2018 pp.

Turkdogan-Aydinol F.I., Yetilmezsoy K., Comez S. y Bayhan H. (2011). Performance evaluation and kinetic modeling of the start-up of a UASB reactor treating municipal wastewater at low temperature. Bioproc. Biosyst. Eng. 34 (2), 153-162. DOI: 10.1007/s00449010-0456-0

Van Haandel A., Kato M.T., Cavalcanti P.F.F. y Florencio L. (2006). Anaerobic reactor design concepts for the treatment of domestic wastewater. Environ. Sci. Bio. $5(1), 21-38$. DOI: $10.1007 / \mathrm{s} 11157-005-4888-y$

Van Lier J.B., Rebac S., Lens P., van Bijnen F., Oude Elferink S.J.W.H., Stams A.J.M. y Lettinga G. (1997). Anaerobic treatment of partly acidified wastewater in a two-stage expanded granular sludge bed (EGSB) system at $8^{\circ} \mathrm{C}$. Water Sci. Technol. 36 (6-7), 317-324. DOI: $10.1016 / \mathrm{S} 0273-1223(97) 00538-6$

Wang W., Ma W., Han H., Li H. y Yuan M. (2011). Thermophilic anaerobic digestion of Lurgi coal gasification wastewater in a UASB reactor. Bioresource Technol. 102 (3), 2441-2447.

DOI: $10.1016 /$ j.biortech.2010.10.140

Wang W. y Han H. (2012). Recovery strategies for tackling the impact of phenolic compounds in a UASB reactor treating coal gasification wastewater. Bioresource Technol. 103 (1), 95-100.

DOI: $10.1016 /$ j.biortech.2011.10.002

Yetilmezsoy K. y Sakar S. (2008). Development of empirical models for performance evaluation of UASB reactors treating poultry manure wastewater under different operational conditions. J. Hazard. Mater. 153 (1-2), 532-543. DOI: 10.1016/j.jhazmat.2007.08.087

Zhang L., Hendrickx T.L.G., Kampman C., Temmink H. y Zeeman G. (2013). Co-digestion to support low temperature anaerobic pretreatment of municipal sewage in a UASB-digester. Bioresource Technol. 148, 560-566. DOI: 10.1016/j.biortech.2013.09.013 